\title{
An Itertive Algorithm with Error Terms for Solving a System of Implicit $n$-Variational Inclusions
}

\author{
Zubair Khan ${ }^{1}$, Syed Shakaib Irfan ${ }^{2}$, M. Firdosh Khan ${ }^{3}$ and P. Shukla ${ }^{1}$ \\ ${ }^{1}$ Department of Mathematics, Faculty of Science, Integral University, Lucknow, India \\ ${ }^{2}$ College of Engineering, Qassim University, Buraidah 51452, Al-Qassim, Saudi Arabia \\ ${ }^{3}$ S. H. Senior Secondary School (Boys), Aligarh Muslim University, Aligarh, U.P., India
}

\begin{abstract}
A new system of implicit $n$-variational inclusions is considered. We propose a new algorithm with error terms for computing the approximate solutions of our system. The convergence of the iterative sequences generated by the iterative algorithm is also discussed. Some special cases are also discussed.
\end{abstract}

Keywords Relaxed Operators, Iterative Algorithm, Convergence Results, Resolvent Operators.

AMS 2010 subject classifications 49J40, 90C33

DOI: $10.19139 /$ soic-2310-5070-705

\section{Historical Perspective and Prelude}

Variational inclusions plays an important role in the generalization of classical variational inequalities. So, we have wide range of applications in many of the fields like non-linear programming, economics, optimization, physics etc. Because of its extensive applications various variational inclusions have been established in recent times. Iterative algorithms have been used by different researchers to solve different classes of variational inequalities and variational inclusion problems. For further information one can see $[6,8,9,10,11,12,13,14,15,19,20,21,24,25,26,28]$ and references therein. A new problem of much more interest which is called as system of variational inequalities (inclusions) were introduced and studied in the literature.

In 2007, Xia and Huang [29] studied variational inclusions with a general $H$-monotone operator in Banach spaces, Ahmad et al. [3, 5, 7] considered resolvent operator technique to explain a system of generalized variational-like inclusions in Banach spaces, Verma [27] established and considered some new systems of variational inequalities in Hilbert spaces and generate some iterative algorithms for approximating the solutions of this system. As a generalization of some variational inequalities, Huang [16, 17] introduced Mann and Ishikawa type perturbed iterative algorithms for generalized non-linear implicit quasi-variational inclusions. Then, Agarwal [1] established sensitivity analysis for the new system of generalized non-linear mixed quasi-variational inclusions.

After that, S. Hussain [18] considered an Ishikawa type iterative algorithm for a generalized variational inclusions. In this paper we study and established a system of $n$-variational inclusions in real Hilbert spaces

\footnotetext{
${ }^{*}$ Correspondence to: Syed Shakaib Irfan (Email: shakaib@qec.edu.sa). College of Engineering, Qassim University, Buraidah 51452, AlQassim, Saudi Arabia.
}

ISSN 2310-5070 (online) ISSN 2311-004X (print)

Copyright $\odot 2020$ International Academic Press 
called a new system of implicit $n$-variational inclusions. By using resolvent operator technique, we propose a $n$-iterative algorithm with error terms for computing the approximate solutions of a new system of implicit $n$ variational inclusions. We also discussed here criteria of convergence. The mathematical approach of our paper is quite different than the methods discussed above.

Let $X$ be a real Hilbert space whose norm and inner product are denoted by $\|$.$\| and \langle.,$.$\rangle respectively, d$ is the metric induced by the norm $\|\|,. 2^{X}$ is the family of all non-empty subsets of $\left.X, C B(X)\right)$ is the closed and bounded subset of $X$ and $\mathcal{H}(.,$.$) is the Hausdorff metric on C B(X)$ defined by

$$
\mathcal{H}(A, B)=\max \left(\sup _{x \in A} d(x, B), \sup _{y \in B} d(A, y)\right)
$$

where $d(x, B)=\inf _{y \in B} d(x, y)$ and $d(A, y)=\inf _{x \in A} d(x, y)$.

We require the following definitions and theorems to achieve the main result of this paper.

Definition 1.1. A mapping $g: X \rightarrow X$ is called

(i) Lipschitz continuous if, there exists a constant $\lambda_{g}>0$ such that

$$
\left\|g\left(x_{1}\right)-g\left(x_{2}\right)\right\| \leq \lambda_{g}\left\|x_{1}-x_{2}\right\|, \text { for all } x_{1}, x_{2} \in X ;
$$

(ii) monotone if,

$$
\left\langle g\left(x_{1}\right)-g\left(x_{2}\right), x_{1}-x_{2}\right\rangle \geq 0, \text { for all } x_{1}, x_{2} \in X ;
$$

(iii) strongly monotone if, there exists a constant $\xi>0$ such that

$$
\left\langle g\left(x_{1}\right)-g\left(x_{2}\right), x_{1}-x_{2}\right\rangle \geq \xi\left\|x_{1}-x_{2}\right\|^{2}, \text { for all } x_{1}, x_{2} \in X ;
$$

(iv) relaxed Lipschitz continuous if, there exists a constant $r>0$ such that

$$
\left\langle g\left(x_{1}\right)-g\left(x_{2}\right), x_{1}-x_{2}\right\rangle \leq-r\left\|x_{1}-x_{2}\right\|^{2}, \text { for all } x_{1}, x_{2} \in X .
$$

Definition 1.2. A mapping $F: X \times X \times X \rightarrow X$ is said to be Lipschitz continuous in the first argument if, there exists a constant $\lambda_{F_{1}}$ such that

$$
\left\|F\left(x_{1}, x_{2}, x_{3}\right)-F\left(y_{1}, x_{2}, x_{3}\right)\right\| \leq \lambda_{F_{1}}\left\|x_{1}-y_{1}\right\|, \text { for all } x_{1}, y_{1}, x_{2}, x_{3} \in X .
$$

In a similar way, we can define the Lipschitz continuity of $F$ in the rest of the arguments.

Definition 1.3. A multivalued mapping $A: X \rightarrow C B(X)$ is said to be $\mathcal{H}$-Lipschitz continuous if, there exists a constant $\delta_{A}$ such that

$$
\mathcal{H}\left(A\left(x_{1}\right), A\left(x_{2}\right)\right) \leq \delta_{A}\left\|x_{1}-x_{2}\right\|, \text { for all } x_{1}, x_{2} \in X .
$$

Definition 1.4 [2]. Let $I: X \rightarrow X$ be an identity mapping and $H: X \rightarrow X$ be a mapping. Then for $\lambda>0$ a multivalued mapping $M: X \rightarrow 2^{X}$ is a said to be $(I-H)$ monotone if, $M$ is monotone, $H$ is relaxed Lipschitz continuous and

$$
[(I-H)+\lambda M](X)=X
$$

Definition 1.5 [2]. Let $H: X \rightarrow X$ be a relaxed Lipschitz continuous mapping and $I: X \rightarrow X$ be an identity mapping. Suppose that $M: X \rightarrow 2^{X}$ is a multivalued, $(I-H)$ - monotone mapping. For $\lambda>0$, relaxed resolvent operator $R_{\lambda, M}^{I-H}: X \rightarrow X$ associated with $I, H$ and $M$ is defined by

$$
R_{\lambda, M}^{I-H}(x)=[(I-H)+\lambda M]^{-1}(x), \text { for all } x \in X,
$$


The following theorems plays an important role in proving our main results which is due to [2].

Theorem 1.1 [2]. Let $H: X \rightarrow X$ be a relaxed Lipschitz continuous mapping, $I: X \rightarrow X$ be an identity mapping and $M: X \rightarrow 2^{X}$ be a mutivalued, $(I-H)$ - monotone mapping. Then for $\lambda>0$, the operator $[(I-H)+\lambda M]^{-1}$ is the single valued.

Theorem 1.2 [2]. Let $I: X \rightarrow X$ be an identity mapping, $H: X \rightarrow X$ be a $r$-relaxed Lipschitz continuous mapping and $M: X \rightarrow 2^{X}$ be a multivalued, $(I-H)$ - monotone mapping. Then the relaxed resolvent operator $R_{\lambda, M}^{I-H}: X \rightarrow X$ is $\frac{1}{1+r}$ Lipschitz continuous. i.e.,

$$
\left\|R_{\lambda, M}^{I-H}\left(x_{1}\right)-R_{\lambda, M}^{I-H}\left(x_{2}\right)\right\| \leq \frac{1}{1+r}\left\|x_{1}-x_{2}\right\|, \forall x_{1}, x_{2} \in X
$$

\section{Formulation of the Problem}

We introduce a new system of implicit $n$-variational inclusions in Hilber spaces and develop an iterative algorithm with error terms for solving this system. For each $i \in\{1,2,3, \ldots n\}$, let $X_{i}$ be a real Hilbert space, let $H_{i}, g_{i}: X_{i} \rightarrow X_{i}, F_{i}, P_{i}: X_{1} \times X_{2} \ldots \times X_{n} \rightarrow X_{i}$ be the single valued mappings and $A_{i 1}, A_{i 2}, \ldots . ., A_{i n}: X_{i} \rightarrow C B\left(X_{i}\right)$ be the multivalued mappings. Let $I_{i}: X_{i} \rightarrow X_{i}$ be the identity mappings and $M_{i}: X_{i} \times X_{i} \rightarrow 2^{X_{i}}$ be the multivalued, $\left(I_{i}-H_{i}\right)$ - monotone mappings. We consider the following system of implicit $n$ variational inclusions (in short, SIVI):

Equivalently

(SIVI)

$$
\left\{\begin{array}{l}
\text { Find }\left(x_{1}, x_{2}, \ldots, x_{n}, u_{11}, u_{12}, u_{13}, \ldots, u_{1 n}, \ldots, u_{n 1}, u_{n 2}, u_{n 3}, \ldots u_{n n}\right) \\
\left(x_{1}, x_{2}, \ldots, x_{n}\right) \in X_{1} \times X_{2} \times \ldots \times X_{n}, \\
u_{i 1} \in A_{i 1}\left(x_{1}\right), u_{i 2} \in A_{i 2}\left(x_{2}\right), \ldots \ldots u_{i n} \in A_{i n}\left(x_{n}\right) \text { such that } \\
0 \in F_{1}\left(x_{1}, x_{2} \ldots, x_{n}\right)+P_{1}\left(u_{11}, u_{12}, \ldots, u_{1 n}\right)+M_{1}\left(g_{1}\left(x_{1}\right), x_{1}\right) \\
0 \in F_{2}\left(x_{1}, x_{2}, \ldots, x_{n}\right)+P_{2}\left(u_{21}, u_{22}, \ldots, u_{2 n}\right)+M_{2}\left(g_{2}\left(x_{2}\right), x_{2}\right) \\
\cdot \\
\cdot \\
\cdot \\
0 \in F_{n}\left(x_{1}, x_{2}, \ldots, x_{n}\right)+P_{n}\left(u_{n 1}, u_{n 2}, \ldots ., u_{n n}\right)+M_{n}\left(g_{n}\left(x_{n}\right), x_{n}\right) .
\end{array}\right.
$$

\section{Special Cases:}

$$
0 \in F_{i}\left(x_{1}, x_{2}, \ldots, x_{n}\right)+P_{i}\left(u_{i 1}, u_{i 2}, \ldots . u_{i n}\right)+M_{i}\left(g_{i}\left(x_{i}\right), x_{i}\right) .
$$

(i) If $F_{1}\left(x_{1}, x_{2}, \ldots, x_{n}\right) \equiv F_{1}\left(x_{1}, x_{2}, x_{3}\right), F_{2}\left(x_{1}, x_{2}, \ldots, x_{n}\right) \equiv F_{2}\left(x_{1}, x_{2}, x_{3}\right), F_{3}\left(x_{1}, x_{2}, \ldots, x_{n}\right) \equiv F_{3}\left(x_{1}, x_{2}, x_{3}\right), F_{4}$, $F_{5}, \ldots, F_{n}=0, \quad P_{1}\left(u_{11}, u_{22}, \ldots, u_{1 n}\right) \quad \equiv P_{1}\left(u_{11}, u_{22}, u_{33}\right), \quad P_{2}\left(u_{21}, u_{22}, \ldots, u_{2 n}\right) \quad \equiv P_{2}\left(u_{21}, u_{22}, u_{23}\right)$, $P_{3}\left(u_{31}, u_{32}, \ldots, u_{3 n}\right) \equiv P_{3}\left(u_{31}, u_{32}, u_{33}\right), P_{4}, P_{5}, \ldots, P_{n} \equiv 0$. Then the problem (SIVI) reduces to find $\left(x_{1}, x_{2}, x_{3}, u_{11}, u_{12}, u_{13}, u_{21}, u_{22}, u_{23}, u_{31}, u_{32}, u_{33}\right)$ such that for each $i \in\{1,2,3\}, \quad x_{1}, x_{2}, x_{3} \in$ $X_{1} \times X_{2} \times X_{3}, u_{i 1} \in A_{i 1}\left(x_{1}\right), u_{i 2} \in A_{i 2}\left(x_{2}\right), u_{i 3} \in A_{i 3}\left(3_{3}\right)$ such that

$$
(\text { SGIVI })\left\{\begin{array}{l}
0 \in F_{1}\left(x_{1}, x_{2}, x_{3}\right)+P_{1}\left(u_{11}, u_{12}, u_{13}\right)+M_{1}\left(g_{1}\left(x_{1}\right), x_{1}\right) \\
0 \in F_{2}\left(x_{1}, x_{2}, x_{3}\right)+P_{2}\left(u_{21}, u_{22}, u_{23}\right)+M_{2}\left(g_{2}\left(x_{2}\right), x_{2}\right) \\
0 \in F_{3}\left(x_{1}, x_{2}, x_{3}\right)+P_{3}\left(u_{31}, u_{32}, u_{33}\right)+M_{3}\left(g_{3}\left(x_{3}\right), x_{3}\right) .
\end{array}\right.
$$

System of generalized implicit variational inclusion (SGIVI) introduced and studied by Ahmad et al [4].

(ii) If $F_{1}\left(x_{1}, x_{2}, x_{3}\right) \equiv F\left(x_{1}, x_{2}\right), F_{2}\left(x_{1}, x_{2}, x_{3}\right) \equiv G\left(x_{1}, x_{2}\right), F_{3} \equiv 0, P_{1}(., ..) \equiv P(.,),. P_{2}(., . ..) \equiv Q(.,$.$) ,$ $P_{3} \equiv 0 . M_{1}\left(g_{1}\left(x_{1}\right), x_{1}\right) \equiv M_{1}\left(g_{1}\left(x_{1}\right)\right), M_{2}\left(g_{2}\left(x_{2}\right), x_{2}\right) \equiv M_{2}\left(g_{2}\left(x_{2}\right)\right), M_{3} \equiv 0$, then the problem (SGIVI) reduces to the problem of finding $\left(x_{1}, x_{2}\right) \in X_{1} \times X_{2}$ such that

$$
(\text { SGMQI }) \quad\left\{\begin{array}{l}
0 \in F\left(x_{1}, x_{2}\right)+P(u, v)+M_{1}\left(g_{1}\left(x_{1}\right)\right) \\
0 \in G\left(x_{1}, x_{2}\right)+Q(u, v)+M_{2}\left(g_{2}\left(x_{2}\right)\right)
\end{array}\right.
$$


which is called the system of generalized mixed quasivariational inclusions with $(H, \eta)$-monotone operators (SGMQI) introduced and studied by Peng and Zhu [23].

(iii) If $P=Q \equiv 0, g_{1}=I_{1}$ (the identity map on $\left.X_{1}\right), g_{2} \equiv I_{2}$ (the identity map on $\left.X_{2}\right), M_{1}\left(g_{1}\left(x_{1}\right)\right)=M_{1}\left(x_{1}\right)$, $M_{2}\left(g_{2}\left(x_{2}\right)\right)=M_{2}\left(x_{2}\right)$ then (SGMQI) reduces to the system of variational inclusion with $(H, \eta)$-monotone operators (SVI) which is to find $(x, y) \in X_{1} \times X_{2}$ such that

$$
\text { (SVI) }\left\{\begin{array}{l}
0 \in F\left(x_{1}, x_{2}\right)+M_{1}\left(x_{1}\right) \\
0 \in G\left(x_{1}, x_{2}\right)+M_{2}\left(x_{2}\right)
\end{array}\right.
$$

Problem (SVI) was introduced and studied by Fang et al [14].

Lemma 2.1. For each $i \in\{1,2, \ldots, n\}$ let $X_{i}$ be a real Hilbert space, $H_{i}, g_{i}: X_{i} \rightarrow X_{i}, F_{i}, P_{i}$ : $X_{1} \times X_{2} \times \ldots \ldots \times X_{n} \rightarrow X_{i}$ be single-valued mappings and $A_{i 1}, A_{i 2}, \ldots, A_{i n}: X_{i} \rightarrow C B\left(X_{i}\right)$ be the multivalued mappings. Let $I_{i}: X_{i} \rightarrow X_{i}$ be the identity mappings and $M_{i}: X_{i} \times X_{i} \rightarrow 2^{X_{i}}$ be the multivalued,, $\left(I_{i}-H_{i}\right)$ monotone mappings. Then, $\left(x_{1}, x_{2}, . ., x_{n}, u_{11}, u_{12}, \ldots . . u_{1 n}, u_{21}, u_{22}, \ldots \ldots . u_{2 n}, . ., u_{n 1}, u_{n 2}, . ., u_{n n}\right)$ with $\left(x_{1}, x_{2}, \ldots, x_{n}\right) \in X_{1} \times X_{2} \times \ldots \times X_{n}, u_{i 1} \in A_{i 1}\left(x_{1}\right), u_{i 2} \in A_{i 2}\left(x_{2}\right), \ldots, u_{i n} \in A_{i n}\left(x_{n}\right)$ is a solution of problem (SIVI), if following equations are satisfied:

$$
g_{i}\left(x_{i}\right)=R_{\lambda_{i}, M_{i}\left(., x_{i}\right)}^{I_{i}-H_{i}}\left[\left(I_{i}-H_{i}\right)\left(g_{i}\left(x_{i}\right)\right)-\lambda_{i} F_{i}\left(x_{1}, x_{2}, \ldots, x_{n}\right)-\lambda_{i} P_{i}\left(u_{i 1}, u_{i 2}, \ldots, u_{i n}\right)\right],
$$

where, $R_{\lambda_{i}, M_{i}\left(., x_{i}\right)}^{I_{i}-H_{i}}=\left[\left(I_{i}-H_{i}\right)+\lambda_{i} M_{i}\left(., x_{i}\right)\right]^{-1}$ are the relaxed resolvent operators and $\lambda_{i}>0$ are constants.

Proof. The proof is a direct consequence of the definition of the relaxed resolvent operator (1.1).

On the basis of the above observations, we propose the following iterative algorithm with error terms for computing the approximate solution of (SIVI).

Algorithm 2.1. For each $i \in\{1,2, \ldots, n\}$, given $x_{i}^{o} \in X_{i}$, take $u_{i 1}^{o} \in A_{i 1}\left(x_{1}^{o}\right), u_{i 2}^{o} \in A_{i 2}\left(x_{2}^{o}\right), \ldots, u_{i n}^{o} \in A_{i n}\left(x_{n}^{o}\right)$ and let

$$
\begin{aligned}
x_{i}^{1}= & \left(1-\mu_{i}\right) x_{i}^{o}+\mu_{i}\left[x_{i}-g_{i}\left(x_{i}^{o}\right)+R_{\lambda_{i}, M_{i}\left(., x_{i}^{o}\right)}^{I_{i}-H_{i}}\left(( I _ { i } - H _ { i } ) \left(g_{i}\left(x_{i}\right)-\lambda_{i} F_{i}\left(x_{1}^{o}, x_{2}^{o}, \ldots, x_{n}^{o}\right)\right.\right.\right. \\
& \left.-\lambda_{i} P_{i}\left(u_{i 1}^{o}, u_{i 2}^{o} \ldots, u_{i n}^{o}\right)\right]+\mu_{i} e_{i}^{o} .
\end{aligned}
$$

Since, $u_{i 1}^{o} \in A_{i 1}\left(x_{1}^{o}\right), u_{i 2}^{o} \in A_{i 2}\left(x_{2}^{o}\right), \ldots \ldots u_{i n}^{o} \in A_{i n}\left(x_{n}^{o}\right)$, by Nadlers theorem, there exist $u_{i 1}^{1} \in A_{i 1}\left(x_{1}^{1}\right), u_{i 2}^{1} \in$ $A_{i 2}\left(x_{2}^{1}\right), \ldots, u_{\text {in }} \in A_{\text {in }}\left(x_{n}^{1}\right)$, such that

$$
\begin{gathered}
\left\|u_{i 1}^{1}-u_{i 1}^{o}\right\| \leq(1+1) \mathcal{H}_{1}\left(A_{i 1}\left(x_{1}^{1}\right), A_{i 1}\left(x_{1}^{o}\right)\right) \\
\left\|u_{i 2}^{1}-u_{i 2}^{o}\right\| \leq(1+1) \mathcal{H}_{2}\left(A_{i 2}\left(x_{2}^{1}\right), A_{i 2}\left(x_{2}^{o}\right)\right) \\
\cdot \\
\cdot \\
\left\|u_{i n}^{1}-u_{i n}^{o}\right\| \leq(1+1) \mathcal{H}_{n}\left(A_{i n}\left(x_{n}^{1}\right), A_{i n}\left(x_{n}^{o}\right)\right) .
\end{gathered}
$$

Again, let

$$
\begin{aligned}
x_{i}^{2}= & \left(1-\mu_{i}\right) x_{i}^{1}+\mu_{i}\left[x_{i}^{1}-g_{i}\left(x_{i}^{1}\right)+R_{\lambda_{i}, M_{i}\left(,, x_{i}^{1}\right)}^{I_{i}-H_{i}}\left(\left(I_{i}-H_{i}\right)\left(g_{i}\left(x_{i}^{1}\right)\right)-\lambda_{i} F_{i}\left(x_{1}^{1}, x_{2}^{1} \ldots, x_{n}^{1}\right)\right.\right. \\
& \left.\left.-\lambda_{i} P_{i}\left(u_{i 1}^{1}, u_{i 2}^{1}, \ldots, u_{i n}^{1}\right)\right)\right]+\mu_{i} e_{i}^{1} .
\end{aligned}
$$

By Nadler's theorem [22], there exists $u_{i 1}^{2} \in A_{i 1}\left(x_{1}^{2}\right), u_{i 2}^{2} \in A_{i 2}\left(x_{2}^{2}\right), \ldots, u_{i n}^{2} \in A_{i n}\left(x_{n}^{2}\right)$ such that,

$$
\left\|u_{i 1}^{2}-u_{i 1}^{1}\right\| \leq\left(1+\frac{1}{2}\right) \mathcal{H}_{1}\left(A_{i 1}\left(x_{1}^{2}\right), A_{i 1}\left(x_{1}^{1}\right)\right)
$$




$$
\begin{gathered}
\left\|u_{i 2}^{2}-u_{i 2}^{1}\right\| \leq\left(1+\frac{1}{2}\right) \mathcal{H}_{2}\left(A_{i 2}\left(x_{2}^{2}\right), A_{i 2}\left(x_{2}^{1}\right)\right) \\
\cdot \\
\qquad u_{i n}^{2}-u_{i n}^{1} \| \leq\left(1+\frac{1}{2}\right) \\
\cdot
\end{gathered}
$$

By induction, we obtain the sequences, $\left\{x_{i}^{n}\right\},\left\{u_{i 2}^{n}\right\}, \ldots,\left\{u_{i n}^{n}\right\}$ satisfying

$$
\begin{gathered}
x_{i}^{n+1}=\left(1-\mu_{i}\right) x_{i}^{n}+\mu_{i}\left[x_{i}^{n}-g_{i}\left(x_{i}^{n}\right)+R_{\lambda_{i}, M_{i}\left(., x_{i}^{n}\right)}^{I_{i}-H_{i}}\left(\left(I_{i}-H_{i}\right)\left(g_{i}\left(x_{i}^{n}\right)\right)\right)-\lambda_{i} F_{i}\left(x_{1}^{n}, x_{2}^{n} \ldots, x_{n}^{n}\right)\right. \\
\left.-\lambda_{i} P_{i}\left(u_{i 1}^{n}, u_{i 2}^{n}, \ldots, u_{i n}^{n}\right)\right]+\mu_{i} e_{i}^{n} \\
\left\|u_{i 1}^{n+1}-u_{i 1}^{n}\right\| \leq\left(1+\frac{1}{n+1}\right) \mathcal{H}_{1}\left(A_{i 1}\left(x_{1}^{n+1}\right), A_{i 1}\left(x_{1}^{n}\right)\right) \\
\left\|u_{i 2}^{n+1}-u_{i 2}^{n}\right\| \leq\left(1+\frac{1}{n+1}\right) \mathcal{H}_{2}\left(A_{i 2}\left(x_{2}^{n+1}\right), A_{i 2}\left(x_{2}^{n}\right)\right) \\
\cdot \\
\cdot \\
\left\|u_{i n}^{n+1}-u_{i n}^{n}\right\| \leq\left(1+\frac{1}{n+1}\right) \mathcal{H}_{n}\left(A_{i n}\left(x_{n}^{n+1}, A_{i n}\left(x_{n}^{n}\right)\right)\right),
\end{gathered}
$$

where $n=0,1,2, \ldots$ for $i=\{1,2, \ldots, n\}, \mu_{i}>0, \lambda_{i}>0$ are constants, $e_{i}^{n} \in X_{i}$ for $n \geq 0$, are errors to take into account a possible inexact computation of the resolvent operator point and $\mathcal{H}_{i}(.,$.$) are the Hausdroff metrics on$ $C B\left(X_{i}\right)$.

\section{Existence and Convergence Analysis}

In this section, we consider those conditions under which the solution of the problem (SIVI) exists and the sequences of the approximate solutions obtained by Algorithm 2.1, converge strongly to the exact solution of the problem (SIVI).

Theorem 3.1. For each $i \in\{1,2, \ldots, n\}$, consider $X_{i}$ is a Hilbert space, $I_{i}: X_{i} \rightarrow X_{i}$ be the identity mappings and $H_{i}, g_{i}: X_{i} \rightarrow X_{i}$ be the single-valued mappings such that $g_{i}$ is $\xi_{i}$-strongly monotone, $\lambda_{g_{i}}$-Lipschitz continuous and $H_{i}$ is $\lambda_{H_{i}}$-Lipschitz continuous, $r_{i}$-relaxed Lipschitz continuous. Suppose that $A_{i 1}, A_{i 2}, \ldots ., A_{i n}: X_{i} \rightarrow C B\left(X_{i}\right)$ are the multivalued mappings such that $A_{i 1}$ is $\delta_{A i_{1}}-D_{1}$-Lipschitz continuous and $A_{i 2}$ is $\delta_{A i_{2}}-D_{2}$-Lipschitz continuous.... $\delta_{A i_{n}}-D_{n}$-Lipschitz continuous, respectively. Let $F_{i}, P_{i}: X_{1} \times X_{2} \times \ldots . . X_{n} \rightarrow X_{i}$ be the singlevalued mappings such that $F_{i}$ 's are Lipschitz continuous in all $n$-arguments with onstants $\lambda_{P_{i 1}}>0, \lambda_{P_{i 2}}>$ $0, \ldots, \lambda_{P_{i n}}>0$, respectively. Suppose that $M_{i}: X_{i} \times X_{i} \rightarrow 2^{X_{i}}$ are the multivalued $\left(I_{i}-H_{i}\right)$-monotone mappings. For $\lambda_{i}>0$ and $h_{i}>0$ assume

$$
\left\|R_{\lambda_{i}, M_{i}(., x)}^{I_{i}-H_{i}}(z)-R_{\lambda_{i}, M_{i}(., y)}^{I_{i}-H_{i}}(z)\right\| \leq h_{i}\|x-y\|, \forall x, y, z \in X_{i},
$$

and

$$
\left\{\begin{array}{l}
k_{i}=1-\mu_{i}+\mu_{i} h_{i}+\mu_{i} \sqrt{1-2 \xi_{i}+\lambda_{g_{i}}^{2}}+\frac{\mu_{i} \lambda_{g_{i}}+\mu_{i} \lambda_{H_{i}} \lambda_{g_{i}}}{1+r_{i}}+\sum_{i=1}^{n} \frac{\mu_{i} \lambda_{j} \lambda_{F_{j i}}}{1+r_{j}}<1 \\
v_{i}=\mu_{i}\left(\sum_{j=1}^{i=n} \frac{\mu_{j} \lambda_{j} \lambda_{P_{j i}} \delta_{A_{j i}}}{1+r_{j}}\right)<1, \\
k_{i}+v_{i}<1, \text { and } 2 \xi_{i}<1+\lambda_{g_{i}}^{2}, \text { for each } i \in\{1,2, \ldots, n\} \\
\sum_{q=1}^{\infty}\left\|e_{1}^{q}-e_{1}^{q-1}\right\| k^{-q}<\infty, \sum_{q=1}^{\infty}\left\|e_{2}^{q}-e_{2}^{q-1}\right\| k^{-q}<\infty, \ldots, \sum_{q=1}^{\infty}\left\|e_{n}^{q}-e_{n}^{q-1}\right\|<\infty \\
\lim _{n \rightarrow \infty} e_{1}^{n}=\lim _{n \rightarrow \infty} e_{2}^{n} \ldots=\lim _{n \rightarrow \infty} e_{n}^{n}=0, \text { for each } k \in(0,1) .
\end{array}\right.
$$


Then the problem (SIVI) admits a solution $\left(x_{1}, x_{2}, \ldots x_{n}, u_{11}, u_{12}, \ldots . u_{1 n}, u_{21}, u_{22}, \ldots . u_{2 n}, u_{31}\right.$, $\left.u_{32}, \ldots . u_{3 n}, u_{n 1}, u_{n 2}, \ldots, u_{n n}\right)$ and iterative sequences $\left\{x_{i}^{n}\right\},\left\{u_{i 1}^{n}\right\},\left\{u_{i 2}^{n}\right\}, \ldots .\left\{u_{i n}^{n}\right\}$ generated by iterative Algorithm 2.1 strongly converge to $x_{i}, u_{i 1}, u_{i 2}, \ldots . u_{i n}$, respectively, for each $i \in\{1,2,3, \ldots, n\}$.

Proof. For each $i \in\{1,2, \ldots, n\}$, let $d_{i}^{n}=\left[\left(I_{i}-H_{i}\right)\left(g_{i}\left(x_{i}^{n}\right)\right)-\lambda_{i} F_{i}\left(x_{1}^{n}, x_{2}^{n} \ldots, x_{n}^{n}\right)-\lambda_{i} P_{i}\left(u_{i_{1}}^{n}, u_{i_{2}}^{n}, \ldots, u_{i_{n}}^{n}\right)\right]$.

Using Algorithm 2.1, condition (3.1) and Theorem 2.2, we have

$$
\begin{aligned}
& \left\|x_{1}^{n+1}-x_{1}^{n}\right\|=\|\left(1-\mu_{i}\right) x_{1}^{n}+\mu_{1}\left[x_{1}^{n}-g_{1}\left(x_{1}^{n}\right)+R_{\lambda_{1}, M_{1}\left(., x_{1}^{n}\right)}^{I_{1}-H_{1}}\left(d_{1}^{n}\right)\right]+\mu_{1} e_{1}^{n}-\left(1-\mu_{1}\right) x_{1}^{n-1} \\
& -\mu_{1}\left[x_{1}^{n-1}-g_{1}\left(x_{1}^{n-1}\right)+R_{\lambda_{1}, M_{1}\left(., x_{1}^{n-1}\right)}^{I_{1}-H_{1}}\left(d_{1}^{n-1}\right)\right]-\mu_{1} e_{1}^{n-1} \| \\
& \leq\left(1-\mu_{1}\right)\left\|x_{1}^{n}-x_{1}^{n-1}\right\|+\mu_{1} \| x_{1}^{n}-x_{1}^{n-1}-\left(g_{1}\left(x_{1}^{n}\right)-g_{1}\left(x_{1}^{n-1}\right) \|\right. \\
& +\mu_{1}\left\|R_{\lambda_{1}, M_{1}\left(., x_{1}^{n}\right)}^{I_{1}-H_{1}}\left(d_{1}^{n}\right)-R_{\lambda_{1}, M_{1}\left(., x_{1}^{n}\right)}^{I_{1}-H_{1}}\left(d_{1}^{n-1}\right)\right\|+\mu_{1} \| R_{\lambda_{1}, M_{1}\left(., x_{1}^{n}\right)}^{I_{1}-H_{1}}\left(d_{1}^{n-1}\right) \\
& -R_{\lambda_{1}, M_{1}\left(., x_{1}^{n}\right)}^{I_{1}-H_{1}}\left(d_{1}^{n-1}\right)+\mu_{1}\left\|e_{1}^{n}-e_{1}^{n-1}\right\| \\
& \leq\left(1-\mu_{1}\right)\left\|x_{1}^{n}-x_{1}^{n-1}\right\|+\mu_{1}\left\|x_{1}^{n}-x_{1}^{n-1}-\left(g_{1}\left(x_{1}^{n}\right)-g_{1}\left(x_{1}^{n-1}\right)\right)\right\| \\
& +\mu_{1}\left\|R_{\lambda_{1}, M_{1}\left(., x_{1}^{n}\right)}^{I_{1}-H_{1}}\left(d_{1}^{n}\right)-R_{\lambda_{1}, M_{1}\left(., x_{1}^{n}\right)}\left(d_{1}^{n-1}\right)\right\| \\
& +\mu_{1}\left\|R_{\lambda_{1}, M_{1}\left(., x_{1}^{n}\right)}^{I_{1}-H_{1}}\left(d_{1}^{n-1}\right)-R_{\lambda_{1}, M_{1}\left(., x_{1}^{n-1}\right)\left(d_{1}^{n-1}\right)}\right\|+\mu_{1}\left\|e_{1}^{n}-e_{1}^{n-1}\right\| \\
& \leq\left(1-\mu_{1}\right)\left\|x_{1}-x_{n-1}\right\|+\mu_{1}\left\|x_{1}^{n}-x_{1}^{n-1}-\left(g_{1}\left(x_{1}^{n}\right)-g_{1}\left(x_{1}^{n-1}\right)\right)\right\| \\
& +\frac{\mu_{1}}{1+r_{1}}\left\|d_{1}^{n}-d_{1}^{n-1}\right\|+\mu_{1} h_{1}\left\|x_{1}^{n}-x_{1}^{n-1}\right\|+\mu_{1}\left\|e_{1}^{n}-e_{1}^{n-1}\right\| \\
& \leq\left(1-\mu_{1}+\mu_{1} h_{1}\right)\left\|x_{1}^{n}-x^{n-1}\right\|+\mu_{1} \| x_{1}^{n}-x_{1}^{n-1}-\left(g_{1}\left(x_{1}^{n}-g_{1}^{n-1}\right) \|\right. \\
& +\frac{\mu_{1}}{1+r_{1}}\left\|d_{1}^{n}-d_{1}^{n-1}\right\|+\mu_{1}\left\|e_{1}^{n}-e_{1}^{n-1}\right\|,
\end{aligned}
$$

and since $g_{1}$ is $\lambda_{g_{1}}$-Lipschitz continuous and $\xi_{1}$ - strongly monotone, we obtain

$$
\begin{aligned}
\left\|x_{1}^{n}-x_{1}^{n-1}-\left(g 1\left(x_{1}^{n}\right)-g_{1}\left(x_{1}^{n-1}\right)\right)\right\|^{2}= & \left\|x_{1}^{n}-x_{1}^{n-1}\right\|^{2}-2\left\langle x_{1}^{n}-x_{1}^{n-1}, g_{1}\left(x_{1}^{n}\right)-g_{1}\left(x_{1}^{n-1}\right)\right\rangle \\
& +\left\|g_{1}\left(x_{1}^{n}\right)-g_{1}\left(x_{1}^{n-1}\right)\right\|^{2} \\
\leq & \left(1-2 \xi_{1}+\lambda_{g_{1}}^{2}\right)\left\|x_{1}^{n}-x_{1}^{n-1}\right\|^{2} .
\end{aligned}
$$

As $g_{1}$ is $\lambda_{g_{1}}$-Lipschitz continuous, $F_{i}$ is Lipschitz continuous in all $n$-arguments with constants $\lambda_{F_{11}}, \lambda_{F_{12}}, \ldots ., \lambda_{F_{1 n}}$, respectively, $P_{1}$ is Lipschitz continuous in all the $n$-arguments with constants $\lambda_{P_{11}}, \lambda_{P_{12}}, \lambda_{P_{13}}, \ldots, \lambda_{P_{1 n}}$ respectively, $A_{11}$ is $\delta_{A_{11}}-D_{1}$ - Lipschitz continuous, $A_{12}$ is $\delta_{A_{12}}-D_{2}$ - Lipschitz continuous, ........ $A_{1 n}$ is $\delta_{A_{1 n}}-D_{n}$ - Lipschitz continuous, respectively, we get

$$
\begin{aligned}
\left\|d_{1}^{n}-d_{1}^{n-1}\right\|= & \|\left(I_{1}-H_{1}\right)\left(g_{1}\left(x_{1}^{n}\right)\right)-\lambda_{1} F_{1}\left(x_{1}^{n}, x_{2}^{n}, \ldots, x_{n}^{n}\right)-\lambda_{1} P_{1}\left(u_{11}^{n}, u_{12}^{n}, \ldots, u_{1 n}^{n}\right) \\
& -\left(I_{1}-H_{1}\right)\left(g_{1}\left(x_{1}^{n-1}\right)+\lambda_{1} F_{1}\left(x_{1}^{n-1}, x_{2}^{n-1}, \ldots, x_{n}^{n-1}\right)\right. \\
& +\lambda_{1} P_{1}\left(u_{11}^{n-1}, u_{12}^{n-1}, \ldots, u_{1 n}^{n-1}\right) \| \\
\leq & \| g_{1}\left(x_{1}^{n}\right)-g_{1}\left(x_{1}^{n-1}\|+\| H_{i}\left(g_{1}\left(x_{1}^{n}\right)\right)-H_{1}\left(g_{1}\left(x_{1}^{n-1}\right)\right) \|\right. \\
& +\lambda_{1}\left\|F_{1}\left(x_{1}^{n}, x_{2}^{n}, \ldots, x_{n}^{n}\right)-F_{1}\left(x_{1}^{n-1}, x_{2}^{n-1}, \ldots, x_{n}^{n-1}\right)\right\| \\
& +\lambda_{1}\left\|P_{1}\left(u_{11}^{n}, u_{12}^{n}, \ldots, u_{1 n}^{n}\right)-P_{1}\left(u_{11}^{n-1}, u_{12}^{n-1}, \ldots, u_{1 n}^{n-1}\right)\right\| \\
\leq & \| g_{1}\left(x_{1}^{n}\right)-g_{1}\left(x_{1}^{n-1}\|+\| H_{1}\left(g_{1}\left(x_{1}^{n}\right)\right)-H_{1}\left(g_{1}\left(x_{1}^{n-1}\right)\right) \|\right. \\
& +\lambda_{1}\left\|F_{1}\left(x_{1}^{n}, x_{2}^{n}, \ldots, x_{n}^{n}\right)-F_{1}\left(x_{1}^{n-1}, x_{2}^{n-1}, \ldots, x_{n}^{n}\right)\right\| \\
& +\lambda_{1}\left\|F_{1}\left(x_{1}^{n-1}, x_{2}^{n}, x_{3}^{n}, \ldots, x_{n}^{n}\right)-F_{1}\left(x_{1}^{n-1}, x_{2}^{n-1}, \ldots, x_{n}^{n}\right)\right\|
\end{aligned}
$$




$$
\begin{aligned}
& +\lambda_{1}\left\|F_{1}\left(x_{1}^{n-1}, x_{2}^{n-1}, \ldots, x_{n}\right)-F_{1}\left(x_{1}^{n-1}, x_{2}^{n-1}, x_{3}^{n-1}, \ldots, x_{n}^{n-1}\right)\right\| \\
& +\lambda_{1}\left\|P_{1}\left(u_{11}^{n}, u_{12}^{n}, \ldots, u_{1 n}^{n}\right)-P_{1}\left(u_{11}^{n-1}, u_{12}^{n}, u_{13}^{n}, \ldots, u_{1 n}^{n}\right)\right\| \\
& +\lambda_{1}\left\|P_{1}\left(u_{11}^{n-1}, u_{12}^{n}, \ldots, u_{1 n}^{n}\right)-P_{1}\left(u_{11}^{n-1}, u_{12}^{n-1}, \ldots, u_{1 n}^{n}\right)\right\| \\
& +\lambda_{1}\left\|P_{1}\left(u_{11}^{n-1}, u_{12}^{n-1}, \ldots, u_{1 n}^{n}\right)-P_{1}\left(u_{11}^{n-1}, u_{12}^{n-1}, \ldots, u_{1 n}^{n-1}\right)\right\| \\
& \leq \lambda_{g_{1}}\left\|x_{1}^{n}-x_{1}^{n-1}\right\|+\lambda_{H_{1}} \lambda_{g_{1}}\left\|x_{1}^{n}-x_{1}^{n-1}\right\|+\lambda_{1} \lambda_{F_{11}}\left\|x_{1}^{n}-x_{1}^{n-1}\right\| \\
& +\lambda_{1} F_{12}\left\|x_{2}^{n}-x_{2}^{n-1}\right\|+\lambda_{1} \lambda_{F_{13}}\left\|x_{3}^{n}-x_{3}^{n-1}\right\|+\ldots .+\lambda_{1} \lambda_{F_{1 n}}\left\|x_{n}^{n}-x_{n}^{n-1}\right\| \\
& +\lambda_{1} \lambda_{P_{11}}\left\|u_{11}^{n}-u_{11}^{n-1}\right\|+\lambda_{1} \lambda_{P_{12}}\left\|u_{12}^{n}-u_{12}^{n-1}\right\| \\
& +\lambda_{1} \lambda_{P_{13}}\left\|u_{13}^{n}-u_{13}^{n-1}\right\|+\ldots .+\lambda_{1} P_{1 n}\left\|u_{1 n}^{n}-u_{1 n}^{n-1}\right\| \\
& \leq \lambda_{g_{1}}\left\|x_{1}^{n}-x_{1}^{n-1}\right\|+\lambda_{H_{1}} \lambda_{g_{1}}\left\|x_{1}^{n}-x_{1}^{n-1}\right\|+\lambda_{1} \lambda_{F_{11}}\left\|x_{1}^{n}-x_{1}^{n-1}\right\| \\
& +\lambda_{1} \lambda_{F_{12}}\left\|x_{2}^{n}-x_{2}^{n-1}\right\|+\left\|\lambda_{1} \lambda_{F_{13}}\right\| x_{3}^{n}-x_{3}^{n-1} \| \\
& +\ldots+\lambda_{1} \lambda_{F_{1 n}}\left\|x_{n}^{n}-x_{n}^{n-1}\right\|+\lambda_{1} \lambda_{P_{11}}\left\|u_{11}^{n}-u_{11}^{n-1}\right\| \\
& +\lambda_{1} \lambda_{P_{12}}\left\|u_{12}^{n}-u_{12}^{n-1}\right\|+\lambda_{1} \lambda_{P_{13}}\left\|u_{13}^{n}-u_{13}^{n-1}\right\| \\
& +\ldots+\lambda_{1} \lambda_{P_{1 n}}\left\|u_{1 n}^{n}-u_{1 n}^{n-1}\right\| \\
& \leq \quad \lambda_{g_{1}}\left\|x_{1}^{n}-x_{1}^{n-1}\right\|+\lambda_{H_{1}} \lambda_{g_{1}}\left\|x_{1}^{n}-x_{1}^{n-1}\right\| \\
& +\lambda_{1} \lambda_{F_{11}}\left\|x_{1}^{n}-x_{1}^{n-1}\right\|+\lambda_{1} \lambda_{F_{12}}\left\|x_{2}^{n}-x_{2}^{n-1}\right\|+\lambda_{1} \lambda_{F_{13}}\left\|x_{3}^{n}-x_{3}^{n-1}\right\| \\
& +\ldots+\lambda_{1} \lambda_{F_{12}}\left\|x_{n}^{n}-x_{n}^{n-1}\right\|+\lambda_{1} \lambda_{P_{11}}\left(1+\frac{1}{n}\right) D_{1}\left(A_{11}\left(x_{1}^{n}\right), A_{11}\left(x_{1}^{n-1}\right)\right. \\
& +\lambda_{1} \lambda_{P_{12}}\left(1+\frac{1}{n}\right) D_{2}\left(A _ { 1 2 } \left(x_{2}^{n}, A_{12}\left(x_{2}^{n-1}\right)\right.\right. \\
& +\lambda_{1} \lambda_{P_{13}}\left(1+\frac{1}{n}\right) D_{3}\left(A_{13}\left(x_{3}^{n}-A_{3}\left(x_{3}^{n-1}\right)\right)\right. \\
& +\ldots+\lambda_{1} \lambda_{P_{1 n}}\left(1+\frac{1}{n}\right) D_{n}\left(A_{1 n}\left(x_{n}^{n}, A_{1 n}\left(x_{n}^{n-1}\right)\right)\right. \\
& \leq \lambda_{g_{1}}\left\|x_{1}^{n}-x_{1}^{n-1}\right\|+\lambda_{H_{1}} \lambda_{g_{1}}\left\|x_{1}^{n}-x_{1}^{n-1}\right\|+\lambda_{1} \lambda_{F_{11}}\left\|x_{1}^{n}-x_{1}^{n-1}\right\| \\
& +\lambda_{1} \lambda_{F_{12}}\left\|x_{2}^{n}-x_{2}^{n-1}\right\|+\lambda_{1} \lambda_{F_{13}}\left\|x_{3}^{n}-x_{3}^{n-1}\right\| \\
& +\ldots+\lambda_{1} \lambda_{F_{1 n}}\left\|x_{n}^{n}-x_{n}^{n-1}\right\|+\lambda_{1} \lambda_{P_{11}} \delta_{A_{11}}\left(1+\frac{1}{n}\right)\left\|x_{1}^{n}-x_{1}^{n-1}\right\| \\
& +\lambda_{1} \lambda_{P_{12}} \delta_{A_{12}}\left(1+\frac{1}{n}\right)\left\|x_{2}^{n}-x_{2}^{n-1}\right\|+\lambda_{1} \lambda_{P_{13}} \delta_{A_{13}}\left(1+\frac{1}{n}\right)\left\|x_{3}^{n}-x_{3}^{n-1}\right\| \\
& +. .+\lambda_{1} \lambda_{P_{1 n}} \delta_{A_{1 n}}\left(1+\frac{1}{n}\right)\left\|x_{n}^{n}-x_{n}^{n-1}\right\| \\
& \leq\left(\lambda_{g_{1}}+\lambda_{1} \lambda_{F_{11}}\right)\left\|x_{1}^{n}-x_{1}^{n-1}\right\|+\left(\lambda_{H_{1}} \lambda_{g_{1}}+\lambda_{1} \lambda_{P_{11}} \delta_{A_{11}}\left(1+\frac{1}{n}\right)\right)\left\|x_{1}^{n}-x_{1}^{n-1}\right\| \\
& +\left(\lambda_{1} \lambda_{F_{12}}+\lambda_{1} \lambda_{P_{12}} \delta_{A_{12}}\left(1+\frac{1}{n}\right)\right)\left\|x_{2}^{n}-x_{2}^{n-1}\right\| \\
& +\left(\lambda_{1} \lambda_{F_{13}}+\lambda_{1} \lambda_{P_{13}} \delta_{A_{13}}\left(1+\frac{1}{n}\right)\right)\left\|x_{3}^{n}-x_{3}^{n-1}\right\| \\
& +\ldots+\left(\lambda_{1} \lambda_{F_{1 n}}+\lambda_{1} \lambda_{P_{1 n}} \delta_{A_{1 n}}\left(1+\frac{1}{n}\right)\right)\left\|x_{n}^{n}-x_{n}^{n-1}\right\| \\
& \leq\left(\lambda_{g_{1}}+\lambda_{1} \lambda_{F_{11}}+\lambda_{H_{1}} \lambda_{g_{1}}+\lambda_{1} \lambda_{P_{11}} \delta_{A_{11}}\left(1+\frac{1}{n}\right)\right)\left\|x_{1}^{n}-x_{1}^{n-1}\right\| \\
& +\left(\lambda_{1} \lambda_{F_{12}}+\lambda_{1} \lambda_{P_{12}} \delta_{A_{12}}\left(1+\frac{1}{n}\right)\right)\left\|x_{2}^{n}-x_{2}^{n-1}\right\|
\end{aligned}
$$




$$
\begin{aligned}
& +\left(\lambda_{1} \lambda_{F_{13}}+\lambda_{1} \lambda_{P_{13}} \delta_{A_{13}}\left(1+\frac{1}{n}\right)\right)\left\|x_{3}^{n}-x_{3}^{n-1}\right\| \\
& +\ldots+\left(\lambda_{1} \lambda_{F_{1 n}}+\lambda_{1} \lambda_{P_{1 n}} \delta_{A_{1 n}}\left(1+\frac{1}{n}\right)\right)\left\|x_{n}^{n}-x_{n}^{n-1}\right\| .
\end{aligned}
$$

Using (3.4) and(3.5), equation (3.3) becomes,

$$
\begin{aligned}
\left\|x_{1}^{n+1}-x_{1}^{n}\right\| \leq & \left(1-\mu_{1}+\mu_{1} h_{1}+\mu_{1} \sqrt{1-2 \xi_{1}+\lambda_{g_{1}}^{2}}\right. \\
& \left.+\frac{\mu_{1}\left(\lambda_{g_{1}}+\lambda_{1} \lambda_{F_{11}}+\lambda_{H_{1}} \lambda_{g_{1}}+\lambda_{1} \lambda_{P_{11}} \delta_{A_{11}}\left(1+\frac{1}{n}\right)\right.}{1+r_{1}}\right)\left\|x_{1}^{n}-x_{1}^{n-1}\right\| \\
& +\frac{\mu_{1}\left(\lambda_{1} \lambda_{F_{12}}+\lambda_{1} \lambda_{P_{12}} \delta_{A_{12}}\left(1+\frac{1}{n}\right)\right)}{1+r_{1}}\left\|x_{2}^{n}-x_{2}^{n-1}\right\| \\
& +\frac{\mu_{1}\left(\lambda_{1} \lambda_{F_{13}}+\lambda_{1} \lambda_{P_{13}} \delta_{A_{13}}\left(1+\frac{1}{n}\right)\right) 1+r_{1}}{1+r_{1}}\left\|x_{3}^{n}-x_{3}^{n-1}\right\|+\mu_{1}\left\|e_{1}^{n}-e_{1}^{n-1}\right\| .
\end{aligned}
$$

Using the same arguments as for (3.6), we get

$$
\begin{aligned}
\left\|x_{2}^{n+1}-x_{2}^{n}\right\| \leq & \left(1-\mu_{2}+\mu_{2} h_{2}+\mu_{2} \sqrt{1-2 \xi_{2}+\lambda_{g_{2}}^{2}}\right. \\
& +\frac{\mu_{2}\left(\lambda_{g_{2}}+\lambda_{2} \lambda_{F_{22}}+\lambda_{H_{2}} \lambda_{g_{2}}+\lambda_{2} \lambda_{P_{22}} \delta_{A_{22}}\left(1+\frac{1}{n}\right)\right)}{1+r_{2}}\left\|x_{2}^{n}-x_{2}^{n-1}\right\| \\
& +\frac{\mu_{2}\left(\lambda_{2} \lambda_{F_{21}}+\lambda_{2} \lambda_{P_{21}} \delta_{A_{21}}\left(1+\frac{1}{n}\right)\right)}{1+r_{2}}\left\|x_{1}^{n}-x_{1}^{n-1}\right\| \\
& +\frac{\mu_{2}\left(\lambda_{2} \lambda_{F_{23}}+\lambda_{2} \lambda_{P_{23}} \delta_{A_{23}}\left(1+\frac{1}{n}\right)\right)}{1+r_{2}}\left\|x_{3}^{n}-x_{3}^{n-1}\right\|+\mu_{2}\left\|e_{2}^{n}-e_{2}^{n-1}\right\| .
\end{aligned}
$$

Using the same arguments as for (3.6), we get

$$
\begin{aligned}
\left\|x_{3}^{n+1}-x_{3}^{n}\right\| \leq & \frac{\mu_{3}\left(\lambda_{3} \lambda_{F_{31}}+\lambda_{3} \lambda_{P_{31}} \delta_{A_{31}}\left(1+\frac{1}{n}\right)\right)}{1+r_{3}}\left\|x_{1}^{n}-x_{1}^{n-1}\right\| \\
& +\frac{\mu_{3}\left(\lambda_{3} \lambda_{F_{32}}+\lambda_{3} \lambda_{P_{32}} \delta_{A_{32}}\left(1+\frac{1}{n}\right)\right)}{1+r_{3}}\left\|x_{2}^{n}-x_{2}^{n-1}\right\| \\
& +\left(1-\mu_{3}+\mu_{3} h_{3}+\mu_{3} \sqrt{1-2 \xi_{3}+\lambda_{g_{3}}^{2}}\right. \\
& +\frac{\mu_{3}\left(\lambda_{g_{3}}+\lambda_{3} \lambda_{F_{33}}+\lambda_{H_{3}} \lambda_{g_{3}}+\lambda_{3} \lambda_{P_{33}} \delta_{A_{33}}\left(1+\frac{1}{n}\right)\right)}{1+r_{3}}\left\|x_{3}^{n}-x_{3}^{n-1}\right\| \\
& +\mu_{3}\left\|e_{3}^{n}-e_{3}^{n-1}\right\| .
\end{aligned}
$$

Using the same arguments as for (3.6), we get

$$
\begin{aligned}
\left\|x_{n}^{n+1}-x_{n}^{n}\right\| \leq & \frac{\mu_{n}\left(\lambda_{n} F_{n 1}+\lambda_{n} \lambda_{P_{n 1}} \delta_{A_{n 1}}\left(1+\frac{1}{n}\right)\right)}{1+r_{n}}\left\|x_{n}^{n}-x_{n}^{n-1}\right\| \\
& +\frac{\mu_{n}\left(\lambda_{n} F_{n 2}+\lambda_{n} \lambda_{P_{n 2}} \delta_{A_{n 2}}\left(1+\frac{1}{n}\right)\right)}{1+r_{n}}\left\|x_{n}^{n}-x_{n}^{n-1}\right\| \\
& +\left(1-\mu_{n}+\mu_{n} h_{n}+\mu_{n} \sqrt{1-2 \xi_{n}+\lambda_{g_{n}}^{2}}\right. \\
& +\frac{\mu_{n}\left(\lambda_{g_{n}}+\lambda_{n} \lambda_{F_{n 3}}+\lambda_{H_{n}} \lambda_{g_{3}}+\lambda_{n} \lambda_{P_{n 3}} \delta_{A_{n 3}}\left(1+\frac{1}{n}\right)\right)}{1+r_{n}}\left\|x_{n}^{n}-x_{n}^{n-1}\right\| \\
& +\mu_{n}\left\|e_{n}^{n}-e_{n}^{n-1}\right\| .
\end{aligned}
$$


Combining (3.6) and (3.9), we get

$$
\begin{aligned}
& \left\|x_{1}^{n+1}-x_{1}^{n}\right\|+\ldots+\left\|x_{n}^{n+1}-x_{n}^{n}\right\| \\
& \leq\left(1-\mu_{1}+\mu_{1} h_{1}+\mu_{1} \sqrt{1-2 \xi_{1}+\lambda_{g_{1}}^{2}}\right. \\
& +\frac{\mu_{1}\left(\lambda_{g_{1}}+\lambda_{1} \lambda_{F_{11}}+\lambda_{H_{1}} \lambda_{g_{1}}+\lambda_{1} \lambda_{P_{11}} \delta_{A_{11}}\left(1+\frac{1}{n}\right)\right)}{1+r_{1}}\left\|x_{1}^{n}-x_{1}^{n-1}\right\| \\
& +\frac{\mu_{1}\left(\lambda_{1} \lambda_{F_{12}}+\lambda_{1} \lambda_{P_{12}} \delta_{A_{12}}\left(1+\frac{1}{n}\right)\right)}{1+r_{1}}\left\|x_{2}^{n}-x_{2}^{n-1}\right\| \\
& +\frac{\mu_{1}\left(\lambda_{1} \lambda_{F_{13}}+\lambda_{1} \lambda_{P_{13}} \delta_{A_{13}}\left(1+\frac{1}{n}\right)\right)}{1+r_{1}}\left\|x_{3}^{n}-x_{3}^{n-1}\right\| \\
& +\mu_{1}\left\|e_{1}^{n}-e_{1}^{n-1}\right\|+\left(1-\mu_{2}+\mu_{2} h_{2}+\mu_{2} \sqrt{1-2 \xi_{2}+\lambda_{g_{2}}^{2}}\right. \\
& +\frac{\mu_{2}\left(\lambda_{g_{2}}+\lambda_{2} \lambda_{F_{22}}+\lambda_{H_{2}} \lambda_{g_{2}}+\lambda_{2} \lambda_{P_{22}} \delta_{A_{22}}\left(1+\frac{1}{n}\right)\right)}{1+r_{2}}\left\|x_{2}^{n}-x_{2}^{n-1}\right\| \\
& +\frac{\mu_{2}\left(\lambda_{2} \lambda_{F_{21}}+\lambda_{2} \lambda_{P_{21}} \delta_{A_{21}}\left(1+\frac{1}{n}\right)\right)}{1+r_{2}}\left\|x_{1}^{n}-x_{1}^{n-1}\right\| \\
& +\frac{\mu_{2}\left(\lambda_{2} \lambda_{F_{23}}+\lambda_{2} \lambda_{P_{23}} \delta_{A_{23}}\left(1+\frac{1}{n}\right)\right)}{1+r_{2}}\left\|x_{3}^{n}-x_{3}^{n-1}\right\| \\
& +\mu_{2}\left\|e_{2}^{n}-e_{2}^{n-1}\right\|+\left(1-\mu_{3}+\mu_{3} h_{3}+\mu_{3} \sqrt{1-2 \xi_{3}+\lambda_{g_{3}}^{2}}\right. \\
& +\frac{\mu_{3}\left(\lambda_{3} \lambda_{F_{33}}+\lambda_{H_{3}} \lambda_{g_{3}}+\lambda_{3} \lambda_{P_{33}} \delta_{A_{33}}\left(1+\frac{1}{n}\right)\right)}{1+r_{3}}\left\|x_{3}^{n}-x_{3}^{n-1}\right\| \\
& +\frac{\mu_{3}\left(\lambda_{3} \lambda_{F_{31}}+\lambda_{3} \lambda_{P_{31}} \delta_{A_{31}}\left(1+\frac{1}{n}\right)\right)}{1+r_{3}}\left\|x_{1}^{n}-x_{1}^{n-1}\right\| \\
& +\frac{\mu_{3}\left(\lambda_{3} \lambda_{F_{32}}+\lambda_{3} \lambda_{P_{32}} \delta_{A_{32}}\left(1+\frac{1}{n}\right)\right)}{1+r_{3}}\left\|x_{2}^{n}-x_{2}^{n-1}+\mu_{3}\right\| e_{3}^{n}-e_{3}^{n-1} \| \\
& +\ldots .+\left(1-\mu_{n}+\mu_{n} h_{n}+\mu_{n} \sqrt{1-2 \xi_{n}+\lambda_{g_{n}}^{2}}\right. \\
& +\frac{\mu_{n}\left(\lambda_{g_{n}}+\lambda_{n} \lambda_{F_{n 3}}+\lambda_{H_{n}} \lambda_{g_{3}}+\lambda_{n} \lambda_{P_{n 3}} \delta_{A_{n 3}}\left(1+\frac{1}{n}\right)\right)}{1+r_{n}}\left\|x_{n}^{n}-x_{n}^{n-1}\right\| \\
& +\frac{\mu_{n}\left(\lambda_{n} \lambda_{F_{n 1}}+\lambda_{n} \lambda_{P_{n 1}} \delta_{A_{n 1}}\left(1+\frac{1}{n}\right)\right)}{1+r_{n}}\left\|x_{n}^{n}-x_{n}^{n-1}\right\| \\
& +\frac{\mu_{n}\left(\lambda_{n} \lambda_{F_{n 2}}+\lambda_{n} \lambda_{P_{n 2}} \delta_{A_{n 2}}\left(1+\frac{1}{n}\right)\right)}{1+r_{n}}\left\|x_{n}^{n}-x_{n}^{n-1}\right\|+\mu_{n}\left\|e_{n}^{n}-e_{n}^{n-1}\right\|,
\end{aligned}
$$

which implies that,

$$
\begin{aligned}
\sum_{i=1}^{n}\left\|x_{i}^{n+1}-x_{i}^{n}\right\| \leq & \sum_{i=1}^{n}\left(1-\mu_{i}+\mu_{i} h_{i}+\mu_{i} \sqrt{1-2 \xi_{i}+\lambda_{g_{i}}^{2}}+\frac{\mu_{i} \lambda_{g_{i}}+\mu_{i} \lambda_{H_{i}} \lambda_{g_{i}}}{1+r_{i}}\right. \\
& \left.+\sum_{j=1}^{n} \frac{\mu_{j} \lambda_{j} F_{j i}}{1+r_{j}}+\sum_{j=1}^{n} \frac{\mu_{j} \lambda_{j} \lambda_{P_{j i}} \delta_{A_{j i}}}{1+r_{j}}\left(1+\frac{1}{n}\right)\right)\left\|x_{i}^{n}-x_{i}^{n+1}\right\| \\
& +\sum_{i=1}^{n} \mu_{i}\left\|e_{i}^{n}-e_{i}^{n-1}\right\| \\
\leq & \sum_{i=1}^{n}\left(k_{i}+v_{i}^{n}\right)\left\|x_{i}^{n}-x_{i}^{n-1}\right\|+\sum_{i=1}^{n} \mu_{i}\left\|e_{i}^{n}-e_{i}^{n-1}\right\|
\end{aligned}
$$


where,

$$
k_{i}=1-\mu_{i}+\mu_{i} h_{i}+\mu_{i} \sqrt{1-2 \xi_{i}+\lambda_{g_{i}}^{2}}+\frac{\mu_{i} \lambda_{g_{i}}+\mu_{i} \lambda_{H_{i}} \lambda_{g_{i}}}{1+r_{i}}+\sum_{j=1}^{n} \frac{\mu_{j} \lambda_{j} \lambda_{F_{j i}}}{1+r_{j}}
$$

and

$$
v_{i}^{n}=\sum_{j=1}^{n} \frac{\mu_{j} \lambda_{j} \lambda_{P_{j i}} \delta_{A_{j i}}}{1+r_{j}}\left(1+\frac{1}{n}\right) .
$$

It follows that from (3.10) that,

$$
\sum_{i=1}^{n}\left\|x_{i}^{n+1}-x_{i}^{n}\right\| \leq \sum_{i=1}^{n} \alpha^{n}\left\|x_{i}^{n}-x_{i}^{n+1}\right\|+\sum_{i=1}^{n} \mu_{i}\left\|e_{i}^{n}-e_{i}^{n-1}\right\|,
$$

where, $\alpha^{n}=\max \left\{k_{1}+v_{1}^{n}, k_{2}+v_{2}^{n}, k_{3}+v_{3}^{n}, \ldots, k_{n}+v_{n}^{n}\right\}, \forall n=1,2,3, \ldots$.

Let $\alpha=\max \left\{k_{1}+v_{1}, k_{2}+v_{2}, k_{3}+v_{3}, \ldots, k_{n}+v_{n}\right\}$

where,

$$
v_{i}=\mu_{i} \sum_{j=1}^{n} \frac{\mu_{j} \lambda_{j} \lambda_{P_{j i}} \delta_{A_{j i}}}{1+r_{j}}, \text { for each } i \in 1,2,3, \ldots, n
$$

then $\alpha_{i}^{n} \rightarrow \alpha$ and $v_{i}^{n} \rightarrow v_{i}$ when $n \rightarrow \infty$ for each $i \in\{1,2,3, \ldots, n\}$.

From condition (3.2), we know that $0<\alpha<1$, and hence there exists $n_{0} \in N$ and $\alpha_{0} \in(\alpha, 1)$ such that $\alpha^{n} \leq \alpha_{0}$ for all $n \geq n_{0}$. Therefore, it follows from (3.10) that,

$$
\sum_{i=1}^{n}\left\|x_{i}^{n+1}-x_{i}^{n}\right\| \leq \sum_{i=1}^{n} \alpha_{n_{0}}\left\|x_{i}^{n}-x_{i}^{n-1}\right\|+\sum_{i=1}^{n} \mu_{i}\left\|e_{i}^{n}-e_{i}^{n-1}\right\|, \forall n \geq n_{0},
$$

which implies that

$$
\sum_{i=1}^{n}\left\|x_{i}^{n+1}-x_{i}^{n}\right\| \leq \sum_{i=1}^{n} \alpha_{0}^{n-n_{0}}\left\|x_{i}^{n_{0}+1}-x_{i}^{n_{0}}\right\|+\sum_{p=1}^{n-n_{0}} \sum_{i=1}^{n} \mu_{i} \alpha_{0}^{p-1} \iota_{i}^{n-(p-1)}, \forall n \geq n_{0},
$$

where $\iota_{i}^{n}=\left\|e_{i}^{n}-e_{i}^{n-1}\right\|$ for all $n \geq n_{0}$. Hence, for any $m \geq n>n_{0}$, we get

$$
\begin{aligned}
\sum_{i=1}^{n}\left\|x_{i}^{m}-x_{i}^{n}\right\| & \leq \sum_{q=n}^{m-1} \sum_{i=1}^{n}\left\|x_{i}^{n_{0}+1}-x_{i}^{n_{0}}\right\|+\sum_{q=n}^{m} \sum_{p=1}^{q-n_{0}} \sum_{i=1}^{n} \mu_{i} \alpha_{0}^{p-1} \iota_{i}^{q-(p-1)} \\
& \leq \sum_{q=n}^{m-1} \sum_{i=1}^{n} \alpha_{0}^{q-n_{0}}\left\|x_{i}^{n_{0}+1}-x_{i}^{n_{0}}\right\|+\sum_{q=n}^{m} \sum_{p=1}^{q-n_{0}} \sum_{i=1}^{n} \mu_{i} \alpha_{0}^{q} \frac{\iota_{i}^{q-(p-1)}}{\alpha_{0}^{q-(p-1)}} .
\end{aligned}
$$

Since,

$$
\sum_{q=1}^{\infty} \iota_{1}^{q} k^{-q}<\infty, \sum_{q=1}^{\infty} \iota_{2}^{q} k^{-q}<\infty, \sum_{q=1}^{\infty} \iota_{3}^{q} k^{-q}<\infty, \ldots, \sum_{q=1}^{\infty} \iota_{n}^{q} k^{-q}<\infty, \forall k \in(0,1) \text { and } \alpha_{0}<1 .
$$

It follows from (3.12), that

$$
\left\|x_{1}^{m}-x_{1}^{n}\right\| \rightarrow 0,\left\|x_{2}^{m}-x_{2}^{n}\right\| \rightarrow 0, \ldots,\left\|x_{n}^{m}-x_{n}^{n}\right\| \rightarrow 0, \text { as } n \rightarrow \infty,
$$

and so $\left\{x_{1}^{n}\right\},\left\{x_{2}^{n}\right\}, \ldots, x_{n}^{n}$ are Cauchy sequences in $X_{1}, X_{2}, \ldots, X_{n}$ respectively. Thus, there exist $x_{1} \in X_{1}, x_{2} \in$ $X_{2} \ldots, x_{n} \in X_{n}$ such that $x_{1}^{n} \rightarrow x_{1}, x_{2}^{n} \rightarrow x, \ldots x_{n}^{n} \rightarrow x_{n}$, when $n \rightarrow \infty$.

Now, we prove that $u_{i_{1}}^{n} \rightarrow u_{i_{1}} \in A_{i_{1}}\left(x_{1}\right), u_{i_{2}}^{n} \rightarrow u_{i_{2}} \in A_{i_{2}}\left(x_{2}\right), \ldots, u_{i_{n}} \rightarrow u_{i_{n}} \in A_{i_{n}}\left(x_{n}\right)$, for each $i \in 1,2, \ldots, n$. It follows from (2.2) - (2.4) and by Lipschitz continuity of $A_{i_{1}}, A_{i_{2}}, \ldots, A_{i_{n}}$

$$
\left\|u_{i_{1}}^{n}-u_{i_{1}}^{n-1}\right\| \leq\left(1+\frac{1}{n+1}\right) \delta_{A_{i_{1}}}\left\|x_{1}^{n}-x_{1}^{n-1}\right\|,
$$




$$
\begin{gathered}
\left\|u_{i_{2}}^{n}-u_{i_{2}}^{n-1}\right\| \leq\left(1+\frac{1}{n+1}\right) \delta_{A_{i_{2}}}\left\|x_{2}^{n}-x_{2}^{n-1}\right\|, \\
\cdot \\
\cdot \cdot \\
\left\|u_{i_{n}}^{n}-u_{i_{n}}^{n-1}\right\| \leq\left(1+\frac{1}{1+n}\right) \delta_{A_{i_{n}}}\left\|x_{n}^{n}-x_{n}^{n-1}\right\| .
\end{gathered}
$$

From (3.13)-(3.15), we know that $\left\{u_{i_{1}}^{n}\right\},\left\{u_{i_{2}}^{n}\right\}, \ldots,\left\{u_{i_{n}}^{n}\right\}$ are Cauchy sequences. Therefore, there exist $u_{i_{1}} \in$ $X_{1}, u_{i_{2}} \in X_{2}, \ldots, u_{i_{n}} \in X_{n}$ such that $u_{i_{1}}^{n} \rightarrow u_{i}, u_{i_{2}}^{n} \rightarrow u_{i_{2}}, \ldots, u_{i_{n}}^{n} \rightarrow u_{i_{n}}$, when $n \rightarrow \infty$.

Further, for each $i \in\{1,2,3, \ldots, n\}$.

$$
\begin{aligned}
d\left(u_{i_{1}}, A_{i_{1}}\left(x_{1}\right)\right) & \leq\left\|u_{i_{1}}-u_{i_{1}}^{n}\right\|+d\left(u_{i_{1}^{n}}, A_{i_{1}}\left(x_{1}\right)\right) \\
& \leq\left\|u_{i_{1}}-u_{i_{1}}^{n}\right\|+\mathcal{H}_{1}\left(A_{i_{1}}\left(x_{1}^{n}\right), A_{i_{1}}\left(x_{1}\right)\right) \\
& \leq\left\|u_{i_{1}}-u_{i_{1}}^{n}\right\|+\left(1+\frac{1}{n+1}\right) \delta_{A_{i_{1}}}\left\|x_{1}^{n}-x_{1}\right\| \rightarrow 0, \text { when } n \rightarrow \infty .
\end{aligned}
$$

Since $A_{i_{1}}$ is closed, we have $u_{i_{1}} \in A_{i_{1}}\left(x_{1}\right)$. Similarly, $u_{i_{2}} \in A_{i_{2}}\left(x_{2}\right), \ldots, u_{i_{n}} \in A_{i_{n}}\left(x_{n}\right)$, respectively. By continuity of the mappings, $g_{i}, H_{i}, F_{i}, P_{i}, R_{\lambda_{i}, M_{i}}^{I_{i}-H_{i}}$ and iterative Algorithm 2.1, we know that $u_{i_{1}}, u_{i_{2}}, \ldots, u_{i_{n}}$ satisfy the following relation:

$$
g_{i}\left(x_{i}\right)=R_{\lambda_{i}, M_{i}\left(., x_{i}\right)}^{I_{i}-H_{i}}\left[\left(I_{i}-H_{i}\right)\left(g_{i}\left(x_{i}\right)\right)-\lambda_{i} F_{i}\left(x_{1}, x_{2}, \ldots, x_{n}\right)-\lambda_{i} P_{i}\left(u_{i_{1}}, u_{i_{2}}, \ldots, u_{i_{n}}\right)\right]
$$

By Lemma $2.1,\left(x_{1}, x_{2}, \ldots, x_{n}, u_{11}, u_{12}, \ldots u_{1 n}, u_{21}, u_{22}, \ldots u_{2 n}, \ldots, u_{n 1}, u_{n 2}, \ldots, u_{n n}\right)$ is a solution of problem (SIVI). This completes the proof.

\section{Conclusion}

In this paper we have considered a new system of implicit $n$-variational inclusions which is more general than many existing system of variational inclusions in the literature. Firstly, we propose a new algorithm with error terms for computing the approximate solutions of our system; and secondly, convergence of the iterative sequences generated by the iterative algorithm is discussed. Some special cases are studied. The implementation and comparison of these methods with other methods is a subject of the future research.

\section{Acknowledgement}

The script communication number(MCN) IU/R and D/2018-MCN 000387, Office of Doctoral Studies and Research, Integral University, Lucknow, India.

\section{REFERENCES}

1. R. P. Agarwal, Multiple positive solutions of singular and non-singular discrete problems via variational methods, J. Nonlin. Anal., vol. 58, pp. 69-73, 2004.

2. I. Ahmad, M. Rahaman, and R. Ahmad, Relaxed resolvent operator for solving a variational inclusion problem, Stat. Optim. Inf. Comput., vol. 4, pp. 183-193, 2016.

3. I. Ahmad, R. Ahmad, and M. Rahaman, Implicit resolvent equation problem in Hilbert spaces, Math. Sci. Lett., vol. 6, no. 2, pp. $1-7,2017$.

4. I. Ahmad, V. N. Mishra, R. Ahmad, and M. Rahaman, An Iterative algorithm for a system of generalized implicit variational inclusions, , Spring Plus, vol. 5, no. 1283, 2016.

5. I. Ahmad, R. Ahmad, and M. Rahaman, A resolvent approach for solving a set-valued variational inclusion problem using weak-RRD set-valued mapping, Korean J. Math., vol. 24, no. 2, pp. 199-213, 2016. 
6. Q. H. Ansari, S. Schaible, and J. C. Yao, System of vector equillibrium problems and its applications, J. Optim. Theory Appl., vol. 107, pp. 547-557, 2000.

7. R. Ahmad, M. Ishtyak, M. Rahaman and I. Ahmad, Graph convergence and generalized Yosida approximation operator with an application, Math. Sci., vol. 11, no. 2, pp. 155-163, 2017.

8. M. Bianchi, Pseudo P-monotone Operators and variational inequalities, Report 6, Istitute di econometria e Matematica per le decisioni economiche, Universita Cattolica del Cuore,Milan, 1993.

9. S. S. Chang, J. K. Kim, and K. H. Kim, On the existence and iterative approximation problems of solutions for set-valued variational inclusions in Banach Spaces, Comput. Math. Appl., vol. 49, pp. 365-374, 2005.

10. Y. J. Cho, Y. P. Fang, and N. J. Huang, Algorithms for systems of nonlinear variational inequalities, J. Korean Math. Soc., vol. 41, pp. 489-499, 2004.

11. G. Cohen, and F. Chaplais, Nested monotony for variatonal inequalities over a product of spaces and convergence of iterative algorithms, J. Optim. Theory Appl., vol. 59, pp. 360-390, 1998.

12. X. P. Ding, Existence and algorithms of solutions for nonlinear mixed variational-like inequalities in Banach spaces, J. Comput. Appl, Math., vol. 157, pp. 419-434, 2003.

13. Y. P. Fang, and N. J. Huang. Existence results for systems of strongly implicit vector variational inequalities, Acta Math. Hung., vol. 103, pp. 265-277, 2004.

14. Y. P. Fang, N. J. Huang, and H. B. Thompson, A new system of variational inclusions with (H, $\eta)$-monotone operators in Hilbert spaces, Comput. Math. Appl., vol. 49, no. 2-3, pp. 365-374, 2005.

15. N. J. Huang, Generalized nonlinear variational inclusions with noncompact valued mapping, Appl. Math. Lett., vol. 9, no. 3, pp. 25-29, 1996.

16. N. J. Huang, A new completely general class of variational inclusions with non compact valued mappings, Comput. Math. Appl., vol. 35, no. 10, pp. 9-14, 1998.

17. N. J. Huang, A new class of generalized variational inclusions involving maximal $\eta$-monotone mappings, Publ. Math., 62, Debrecen, 2003.

18. S. Hussain, An Ishikawa type ierative algorithm for a generalised variational inclusions, J. Inter. Math. Forum, vol. 25, pp. 12211228,2009

19. S. S. Irfan, Generalized Variational-like Inclusion in Fuzzy Environment with S-Proximal Operator J. Math. Anal., vol. 10, no. 3 , pp. 89-99, 2019.

20. J. K. Kim, and D. S. Kim, A new system of generalized nonlinear mixed variational inequalities in Hilbert spaces, J. Korean Math. Soc., vol. 11, no. 1, pp. 203-210, 2004.

21. H. Y. Lan, J. H. Kim, and Y. J. Cho, On a new system of nonlinear monotone multivalued variational inclusions, J. Math. Anal. Appl., vol. 327, pp. 481-493, 2007.

22. S. B. Nadler, Multivalued contraction mappings, Pacific J. Math., vol. 475-488, 1969

23. J. W. Peng, and D. Zhu, A new system of generalised mixed quasivariational inclusions with ( $H, \eta)$-monotone operators, J. Math. Anal. Appl., vol. 327, pp. 175-187, 2007.

24. M. Rahaman, R. Ahmad, M. Dilshad, and I. Ahmad, Relaxed $\eta$-proximal operator for solving a variational-like inclusion problem, Math. Model. Anal., vol. 20 (6), pp. 819-835, 2015.

25. M. Rahaman, Y.C. Liou, R. Ahmad, and I. Ahmad, Convergence theorems for split equality generalized mixed equilibrium problems for demi-contractive mappings, J. Inequal. Appl., vol. 2015 (1), pp. 1-25, 2015.

26. N. K. Sahu, N. K. Mahato, and R. N. Mohapatra, System of nonlinear variational inclusion problems with $(A, \eta)$-maximal monotonicity in Banach spaces, Stat Optim Inf Comput, vol. 5, pp. 244-261, 2017.

27. R. U. Verma, Nonlinear variational and constrained hemivariational inequalities involving relaxed operators, J. Appl. Math. Mech., vol. 77, no. 7, pp. 387-391, 1999.

28. J. $\mathrm{Wu}$, and $\mathrm{G}$. Yu, On the convergence and $O(1 / N)$ complexity of a class of nonlinear proximal point algorithms for monotonic variational inequalities, Stat Optim Inf Comput, vol. 2, pp. 105-113, 2014.

29. F. Q. Xia, and N. J. Huang, Algorithm for solving a new class of general mixed variational inequalities in Banach spaces, J. Comput. Math. App., vol. 220, pp. 632-642, 2007. 